\title{
Les sciences régionales au Québec, une discipline et une école qui s'accrochent à la réalité
}

\author{
Bernard Vermot-Desroches, UQTR \\ «L’École Québécoise de Sciences Régionales »
}

\section{Introduction}

Une école évoque la stabilité et caractérise un enseignement particulier. Peut-on parler d'école québécoise des sciences régionales? Quels en seraient ses particularités et l'enseignement transféré ? La réponse est souple et dépend de bien des facteurs. Cet article livre seulement une perception sur le sujet. Une perception certes très partagée mais non universelle.

Lorsque l'on évoque une école de sciences régionales, on peut tout simplement se situer au niveau décisionnel des gouvernements où cette discipline est à la fois théorisée puis mise en pratique par des fonctionnaires. Le niveau peut être beaucoup plus intellectuel si les théoriciens et les chercheurs développent leurs réflexions en des schémas qui vont s'agréger peu à peu en courants de pensée dominants. Il existe enfin le niveau de «la vraie vie». Celui où la population concernée crie fort et clair ses besoins et ses idées pour influencer les spécialistes des deux niveaux précédents.

À la base, une école de sciences régionales implique un regroupement d'experts qui à partir d'objectifs généraux développent des schémas de pensées originaux qui vont se tenir dans la continuité. Ce qui permet alors, à terme et de façon additive, le transfert et la croissance de la discipline en augmentant sa reconnaissance auprès du grand public.

Une école de sciences régionales implique un regroupement d'experts qui à partir d'objectifs généraux développent des schémas de pensées originaux qui vont se tenir dans la continuité

Le passé récent permet de mieux saisir ces éléments. Effectivement les sciences régionales ont émergé en tant que discipline peu de temps après les premières grandes pratiques de développement régional imposées au Québec dans les années '60. À quelques exceptions près, l'école des sciences régionales a toujours suivi les pratiques concrètes, elle ne les a jamais précédées. Elle n'a donc pas grande influence sur la politique et bien sûr encore moins sur le vécu de la population.

Nous allons voir à travers cette histoire récente que les sciences régionales au Québec ont presque toujours été des sciences appliquées dans un esprit thérapeutique. L.E. Hamelin (2006) parle de régiologie. 


\section{Une évolution historique révélatrice}

Bien sûr, on peut toujours remonter très loin dans le temps et s'intéresser aux premières prémices qui sont perceptibles dans ces études et analyses régionales. L'histoire du Québec comme celle du Canada a imposé très tôt des réflexions sur le sujet. La présence des différentes communautés linguistiques et des différentes ethnies imposait de soi des découpages territoriaux qui parfois se faisaient au bout des fusils mais qui le plus souvent imposaient des négociations argumentées et réfléchies.

Ces côtés très anciens de la science régionale ne sont pas abordés ici, en revanche les temps modernes et l'époque contemporaine sont clairement révélateurs.

\subsection{Les temps modernes de notre science régionale, la période du BAEQ où une allure « régiologique » est déjà donnée}

Les régions et leurs développements émergent d'une pensée réfléchie et argumentée au début des années '60. La Grande noirceur de la période de Maurice Duplessis est terminée, une révolution dite tranquille s'anime à tous les niveaux de la société québécoise. Aux niveaux culturel et religieux bien sûr mais aussi à bien d'autres niveaux plus cachés ou moins évidents. À partir de 1960, l'appareil administratif se transforme rapidement et radicalement. Les ministères ne sont plus menés par un seul membre de l'UnionNationale, généralement un ami ou un «sousfifre » du Premier ministre Duplessis. Ils sont menés par une personne plus compétente qui tente de s'entourer de hauts fonctionnaires qui se veulent beaucoup plus efficaces.

Un vent de liberté et de renouveau soufflait sur cet appareil administratif qui vivait sa première jeunesse. Ces hauts fonctionnaires se sentaient imbus d'un pouvoir qui ne pouvait qu'apporter le bien-être aux citoyens. Tout est subitement bouleversé. Les appareils financiers et fiscaux sont redessinés, les systèmes éducatifs de la maternelle à l'université sont rehaussés pour le meilleur. Le Rapport Parent (1963) qui a donné naissance au Ministère de l'éducation du Québec en est l'exemple le plus connu. La Santé et le système hospitalier connaissent aussi un renouveau bienfaiteur.

\subsubsection{La période du BAEQ}

Les régions, ces régions qui ne sont pas Montréal, ont besoin d'aide. En 1960, selon les administrateurs, elles ne bénéficiaient pas nécessairement du bien-être qui leur était dû. Certaines étaient moins développées parce qu'elles manquaient d'efficacité économique, toutes les choses n'étaient pas nécessairement à la bonne place au bon moment... Les fonctionnaires - penseurs avaient été formés en ce sens dans les grandes écoles américaines ou européennes du moment.

\section{Le gouvernement du temps, celui de Jean Lesage (parti libéral) créa le fameux BAEQ en 1963}

Le gouvernement du temps, celui de Jean Lesage (parti libéral) créa le fameux BAEQ en 1963 par la fusion de deux organismes déjà bien en place, le Conseil d'orientation économique du Bas-Saint-Laurent et le Conseil régional d'expansion économique de la Gaspésie et des Îles-de-La-Madeleine. Ce fameux BAEQ se voulait crédible, de jeunes chercheurs comme Guy Coulombe ou JeanClaude Lebel ${ }^{1}$ amenaient beaucoup de dynamisme. Georges-Henri Dubé qui dirigeait ce BAEQ était un notaire reconnu et apprécié des gens de la place. Il voulait toujours respecter certains critères sociaux dans le développement de la région. Plusieurs 
sociologues intervenaient directement dans cette opération.

Il en ressort cependant qu'une douzaine de villages ont été fermés avec leur paroisses. Les habitants ont été alors dispersés vers Matane et la côte gaspésienne. Les "Opérations dignité » ont mis sur pied de nombreux regroupements de la population dans différents secteurs, notamment les secteurs qui touchaient à la forêt.

Cette première époque des temps modernes du développement régional fait déjà ressortir les trois niveaux d'interventions évoqués en introduction. 1- Le gouvernement démarre et anime l'opération. 2- Les chercheurs bardés de nouvelles théories appuient ou adaptent cette opération. 3- Une bonne partie de la population du moment approuve. Seules les familles arrachées à leur paroisse d'origine se sont retrouvées dans le malheur que peut imposer ce non-respect de l'appartenance.

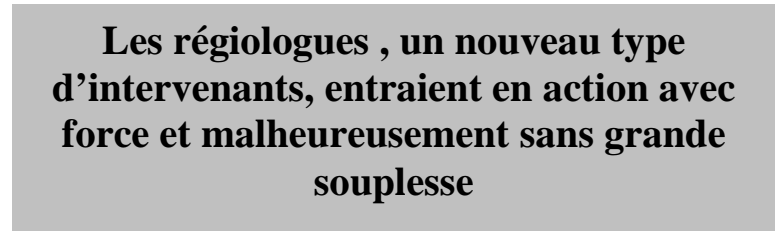

Il apparait donc clairement que cette opération BAEQ était une opération préparée et réfléchie qui s'imposait comme un médecin pouvait imposer un traitement à son patient. Les régiologues, un nouveau type d'intervenants, entraient en action avec force et malheureusement sans grande souplesse. Certaines critiques très virulentes ont été jusqu'à comparer ces interventions du BAEQ à celles que se pratiquaient dans l'URSS d'alors. Quarante-cinq ans plus tard, il semble toujours difficile de voir dans ces prémices du développement régional québécois une esquisse, même discrète, d'une quelconque école de pensée académique. Le BAEQ fut une activité qui dura quatre années et qui s'inscrit davantage dans un ensemble d'actions gouvernementales ad hoc. On n'y retrouve pas la naissance d'un courant de pensée qui pourrait s'instaurer de façon durable sous la forme d'une école. Les sciences régionales balbutiaient, il n'y avait pas de place pour une pensée durable et structurée.

\subsubsection{D'autres mouvements contemporains au $B A E Q$}

Le BAEQ a connu une drôle de célébrité entre 1963 et 1967, cependant cette expérience n'était pas unique, ni au Québec ni au Canada. La fameuse loi ARDA (Aménagement Rural et Développement de l'Agriculture), donnait en 1963 un ton général aux régions du pays. Elle permettait et encourageait la naissance de programmes similaires à celui du BAEQ.

Il faut signaler aussi que dès le début des années '60, le gouvernement québécois créait le Conseil d'Orientation Économique du Québec (COEQ en 1961). Ce conseil devait présenter un plan d'aménagement économique le plus rationnel et le plus efficace possible, tant au niveau des ressources matérielles qu'au niveau des ressources humaines. Cet organisme composé de 15 experts n'avait que des recommandations à formuler. Aboli en 1968 il sera aussitôt remplacé par un successeur important et imposant, l'OPDQ.

D'autres activités voient le jour au niveau des régions dans la foulée de la révolution tranquille. Le tourisme est un des secteurs qui a été rapidement priorisé : le Parc du Forillon, ceux du Mont-Saint-Pierre, de Percé, de Carleton et $\mathrm{du}$ Bic connaissent des développements remarquables et très positifs pour toute la région de la Gaspésie.

Le courant de pensée dominant dans toutes ces activités se dirige vers ce qu'il est convenu d'appeler la ruralité. Les forces viennent du milieu et directement des régions concernées. 
Cependant il n'y a toujours pas d'école qui prenne naissance; cette ruralité n'est pas naturelle ni volontaire, elle est surtout commandée et imposée par des fonctionnaires technocrates souvent très passionnés mais néophytes.

\subsubsection{Un premier retour de balancier, l'OPDQ et le Rapport HMR}

Une nouvelle idée s'impose parfois par des formulations extrêmes qui vont dans le sens opposé à celui que donnait le courant d'idée précédent. En sciences régionales, ce phénomène est très fort. Ainsi à partir de 1967, la Révolution tranquille faisait place à des changements peut-être moins radicaux mais dont l'importance est encore présente dans bien des esprits. Le BAEQ est définitivement fermé, cette expérience, d'un coût initial et direct de plus de 5 millions de dollars de l'époque, se révèle de plus en plus discutable et de nouvelles théories du développement régional émergent tranquillement des travaux d'économistes célèbres comme François Perroux $^{2}$ ou Walter Isard ${ }^{3}$.

Il s'agit principalement de la théorie dite des pôles de croissance, une théorie qui ne cherche plus à aider directement les régions touchées par la pauvreté ou le déclin mais qui vise au développement de tout un territoire à partir des zones particulières qui économiquement sont déjà actives ou jouissent d'un potentiel prometteur capable d'entraîner tout le territoire vers le développement recherché. Déjà en 1967 le MIC (Ministère de l'Industrie et du Commerce) proposait une étude sur les pôles d'attraction et leurs zones d'influence (Gravel, 1999).

L'OPDQ, issu du COEQ disparu en 1968, est un organisme qui prend officiellement les choses en main. Il n'est plus question de ne faire que de simples recommandations au gouvernement mais bien «d'administrer les politiques de développement régional ainsi que de coordonner les interventions de l'État dans les régions ${ }^{4}$. Cet OPDQ ressemblait à une sorte de DATAR (Délégation à l'aménagement $\mathrm{du}$ territoire et à l'action régionale) française adaptée aux réalités régionales du Québec ${ }^{5}$. Les CRD naissent en même temps et sont financés par ce nouvel OPDQ. Ils sont en charge de provoquer et surtout de dynamiser les activités socioéconomiques de chaque région.

En 1970, le MEER conjointement avec ce nouvel OPDQ avait demandé à trois analystes, Higgins, Martin et Reynaud ${ }^{6}$ de définir des objectifs de développement en tenant compte d'un aménagement optimal du territoire. Cette idée d'aménagement du territoire venait directement des nouvelles politiques françaises de développement régional valorisées et imposées au cours des années '60.

\section{On peut promouvoir le développement régional à partir de la région elle-même ou à partir d'un ensemble d'actions extérieures à la région}

On peut promouvoir le développement régional à partir de la région elle-même ou à partir d'un ensemble d'actions extérieures à la région. Higgins, Martin et Reynaud ont mis en valeur cette deuxième approche «à la Perroux » avec une conviction telle que le gouvernement a changé complètement son fusil d'épaule dans ce domaine. Polèse et Shearmur (2002) font bien ressortir l'aspect extrême de ce mouvement de balancier. L'idée de base de ce rapport était pertinente et la théorie des pôles de croissance se justifiait par une logique évidente. On encourage donc le dynamisme économique en plaçant ces pôles aux premières lignes et en les valorisant très fortement. 
Une nouvelle fois, on imposait une prescription aux "patientes » que sont les régions. Selon ces trois analystes, seule Montréal pouvait aider, voire sauver les régions. Qu'il s'agisse d'une région très fortement liée à la capitale comme la ville de Laval ou d'une région périphérique et très éloignée comme la Gaspésie, le remède restait le même et son efficacité semblait garantie. Ces trois auteurs en bon régiologues ont fortement suscité le gouvernement à agir selon cette théorie et la population concernée, surtout celle de Montréal, est bien naturellement entrée dans ce nouveau jeu.

Un retour de balancier qui ne dessine pas vraiment une école de pensée, il s'agit davantage d'une confirmation de cette volonté gouvernementale de diriger un développement planifié. La notion de pôle de développement s'inscrit dans un nouveau courant de pensée sur le développement régional mais ne définit toujours pas une véritable école dans ce domaine. Clermont Dugas parle de «velléité de développement planifié »?

\subsubsection{Un autre retour de balancier : les Sommets régionaux inachevés}

$\mathrm{Au}$ tournant des années 80, «l'effet Montréal » apparaît de moins en moins évident vis-à-vis du développement de bien des régions surtout celles dites périphériques comme l'Abitibi ou la Gaspésie. Bien sûr la conjoncture économique du moment n'est favorable à aucun développement mais l'idée de redonner directement aux régions une plus grande autonomie dans leur développement renaît. Un premier geste concret apparaît dans la création des MRC (Municipalité Régionales de comté) à partir de 1981. Les régions administratives au nombre de dix passent à 16 après que l'on eut redéfini en sous régions la grande région dominante de Montréal.
Une autre idée faisait son chemin, il s'agissait d'évaluer la situation particulière des grandes régions administratives et de promouvoir la concertation à partir de sommets économiques régionaux (Gendron, 1983). Les aspects plus locaux étaient mis sur la scène et le balancier opérait un retour complet, presque extrême. Plusieurs régions profiteront au mieux de cette ouverture, malheureusement d'autres régions ne connaîtront pas cette période récréative, faute de temps et de moyens administratifs.

L'OPDQ devenue vieille et onéreuse suivait au mieux ces différentes activités mais perdait de plus en plus de terrain. Elle disparaîtra totalement en 1991, ses compétences et responsabilités sont réparties alors vers les différents ministères concernés.

Aucune école académique ne naît de cette période, les résolutions et surtout les décisions et les actions régionales vont dans un sens assez clair. La notion de local prend naissance, mais les intellectuels se contentent simplement de rapporter ce que le gouvernement tente d'imposer. Différents rapports comme celui du ministre Picotte ou ceux d'intellectuels universitaires s'imposent seulement en comptes-rendus de bilans ou en recommandations.

\subsection{L'époque contemporaine, le XXI ${ }^{\text {ième }}$ siècle}

Durant les années 90, les gouvernements tant fédéral que provincial tergiversent quant à l'allure que pourrait prendre leurs politiques régionales. Le rapport Picotte (1992) du gouvernement provincial propose une réforme qui tente de fixer les acquis qui commençaient à émerger des sommets régionaux menés au cours de la décennie précédente. Cette réforme donne plus de responsabilités aux organismes régionaux et locaux et amorce un changement dans les concertations entre les
Aucune école académique ne naît de cette période 
différents niveaux de pouvoir. Il s'agit cependant d'une réforme trop ambitieuse, elle visait par exemple l'élimination complète des disparités régionales. En conséquence, sa crédibilité avait parfois du mal à sortir des bureaux du gouvernement.

Le $X X X^{\text {ième }}$ siècle se termine sur une note positive par la création en 1997 d'un Ministère des régions. Sa dénomination et ses structures vont changer aussi souvent que ses titulaires. Cependant, cet effort a été souvent souligné autant par les gens des milieux ruraux que par ceux des milieux urbains.

\section{Le $\mathrm{XX}^{\text {ième }}$ siècle se termine sur une note positive par la création en 1997 d'un Ministère des régions}

\subsubsection{La fin d'un entre-deux}

Entre 1997 et 2002 le Québec se situe dans une forme de latence qui émergea peu après le Rapport Picote. Les régions ne sont évoquées que lorsqu'elles ont des difficultés ou des besoins particuliers. Elles ne sont plus dans le décor immédiat des instances gouvernementales. Bien des choses ont été tentées au cours de ces années sans qu'il n'y ait eu de véritables changements positifs pour les populations concernées.

Une assez longue période riche en politiques diverses mais sans grands résultats tangibles. Pourtant autant le gouvernement Bouchard que le gouvernement Landry avait des velléités annoncées. Les analystes scientifiques se sont aussi mis en veille.

\subsubsection{Les «Fusions - défusions » et le "Rendez-vous national des régions »}

Au tournant du siècle actuel, les actions réelles $\mathrm{du}$ gouvernement concernent davantage cette nouvelle notion de «territoire » vis-à-vis de laquelle l'appartenance est plus claire: une notion déjà ancienne si on se réfère aux géographes. Une notion qui en géographie faisait toujours appel dans sa définition aux termes d'appartenance, que celle-ci soit physique (exemple du relief) ou humaine (exemple de la culture). Bien sûr les économistes se sont empressés de généraliser cette notion d'appartenance que l'on retrouve chez les résidants producteurs et consommateurs.

Une première action a fait couler beaucoup d'encre, il s'agissait d'opérer un regroupement rationnel de nombreuses municipalités du Québec. Contre vents et marées, en 2002, le gouvernement a forcé la fusion parfois trop radicale de ces municipalités. Un changement de gouvernement en 2003 a renversé le mouvement des fusions en un mouvement de défusions.

Une seconde action, bien publicisée et rassemblant beaucoup de monde, a eu lieu en novembre 2002 à Québec, le Rendez-vous National des Régions. Peu avant cette date, le gouvernement provincial qui avait tant parlé des difficultés que rencontraient certaines régions se faisait de plus en plus interrogé sur son inaction. Conscient de cette lacune et des élections qui approchaient (avril 2003), Bernard Landry a élaboré ce rendez-vous qui au départ voulait s'imposer comme un sommet. L'objectif général visait l'élaboration d'un bilan global et d'éventuelles stratégies qui pouvaient sortir de leur marasme certaines entités régionales. En fait, durant dix jours, de nombreuses concertations ont eu lieu tant au niveau de la Province qu'au niveau des régions et des localités mais rien de très substantiel et de durable n'a pu réellement se dégager de ces rencontres. L'évènement était très visible et les médias étaient presque aussi nombreux que les intervenants. Les élections du printemps 2003 ont sorti Bernard Landry et son parti de l'arène politique. Ce rendez-vous 
des régions et ses aboutissements se sont depuis mis en veilleuse. de mettre toujours en priorité l'amélioration de la qualité de vie rurale ${ }^{8}$.

Les sciences régio-nales évoluent et se

Ces deux grandes activités qui traitaient de problèmes régionaux et territoriaux furent elles aussi des activités fondamentalement politiques et peu orientées vers les
Ces deux grandes activités qui traitaient de problèmes régionaux et territoriaux furent elles aussi des activités fondamentalement politiques et peu orientées vers les réels besoins des populations urbaines ou rurales diversifient dans cette foulée. Des publications se font dans des secteurs de plus en plus précis et peuvent même créer de nouveaux liens avec des disciplines plus réels besoins des populations urbaines ou rurales. Rarement les experts ont pu donner un avis autrement qu'à travers les médias, ils n'ont pu que constater et prendre note de ces nouvelles velléités du gouvernement.

Là non plus on ne peut trouver de la part des analystes et des chercheurs autre chose que quelques courants épars d'idées qui se sont vérifiés, infirmés ou modifiés. Il n'y a toujours pas d'école traditionnelle à l'horizon.

\subsubsection{Et maintenant}

Les actions gouvernementales se complexifient de plus en plus. Les instances régionales du gouvernement du Québec se multiplient (CLD, SDÉR, CRÉ, etc.) et les mesures de ce gouvernement vont dans bien des sens. Certains programmes s'intéressent à l'accroissement de la valeur ajoutée, d'autres à la croissance du secteur des ressources naturelles et d'autres veulent faciliter le développement des PME. Les régions ressources sont parfois privilégiées et des secteurs particuliers comme ceux de la forêt sont souvent mis de l'avant. Notons enfin ce souci de plus en plus présent du gouvernement

éloignées ou connexes comme l'histoire, la géographie, la socio-économie, l'éducation, la santé ou même la médicométrie ${ }^{9}$. Le balancier que nous évoquions plus haut n'oscille plus entre les deux extrêmes mais semble vouloir se stabiliser vers un juste milieu. Le Rendezvous national de régions de 2002 le démontre assez bien.

L'aspect régiologie des sciences régionales est de plus en plus présent. Les plus récents articles de notre Revue Canadienne des sciences régionale et même ceux de la Revue d'économie régionale et urbaine (RERU) sont définitivement tournés vers le concret de la vraie vie. Les scientifiques régionaux deviennent de plus en plus des régiologues. Bien du monde trouve que ceci est conforme à l'évolution moderne de bien d'autres disciplines qui veulent devenir plus directement utiles. Les sciences pures peuvent en souffrir à court terme mais à long terme, elles sauront bénéficier de l'essentiel de ces pratiques de terrain. La partie suivante part de cette observation.

Les scientifiques régionaux deviennent de plus en plus des régiologues

\section{Les leçons de l'histoire, des pensées et des actions indécises}

Une condition nécessaire mais non suffisante pour qu'une école puisse voir le jour réside dans l'existence d'une élite de base dans le domaine considéré. Oui, le Québec possède une telle élite. Celle-ci est omniprésente autant dans les milieux universitaires et 
intellectuels que dans les institutions publiques comme les ministères qui regroupent les différents acteurs. Peut-être y a-t-il trop d'interdépendance entre ces deux grands blocs que sont les intellectuels purs et les intellectuels plus appliqués ou administratifs.

2.1 Une élite intellectuelle presque liée à l'attraction des pouvoirs gouvernementaux

L'histoire de la science régionale au Québec commence véritablement en 1960 en même temps que la révolution tranquille. Notre revue très succincte de ces 50 dernières années dans ce domaine montre que les activités gouvernementales qui ont marqué cette longue période connaissent des orientations très diverses et se promènent facilement d'un bord ou de l'autre. Certaines décisions, certaines politiques se contredisent, d'autres se sont révélées inutiles. Cependant on a su très bien rétablir des faiblesses et des problèmes comme ceux des disparités ou de la pauvreté très localisée.

L'époque du BAEQ est une période dont les éléments négatifs sont très faciles à faire ressortir. Cependant les «Opérations dignité» ont forgé des regroupements qui ont eu un effet remarquable dans les prises de conscience; cet effet est encore présent dans certaines localités et certains secteurs. Si aucune école n'en est née, bien des gens et bien des intellectuels en ont tiré des leçons qui se transfèrent encore de nos jours.

L'époque du début de l'OPDQ et du rapport HMR a eu des effets similaires encore plus marqués, l'introduction de cette notion de pôles de croissance a placé au début des années 70 les régions face à Montréal. Les intellectuels se campaient clairement d'un bord ou de l'autre. Là aussi les leçons se sont transférées et se transfèrent encore de nos jours.
Les époques qui ont suivi font ressortir tout aussi clairement ce clivage parfois constructif mais souvent réducteur de ces intellectuels de la science régionale.

Actuellement, les analystes réfléchissent dans les coulisses mais très rapidement - notre milieu étant restreint - ces penseurs sont identifiés. Ils sont presque immanquablement amenés à plonger sur la scène du réel et cela les freine radicalement. On va leur demander de formuler des avis et des recommandations au travers de nombreux rapports qui pour la plupart finiront sur les tablettes. Ces analystes tout à fait libres se transforment alors en analystes influents et engagés de fait. De penseurs et d'intellectuels qu'ils étaient, ils deviennent conseillers et sont parfois questionnés comme des devins voire des guérisseurs, ils sont devenus régiologues sans le vouloir. Naturellement ils vont devoir subir et suivre les cheminements changeants des gouvernements.

\section{Cette structure nécessaire qui formerait les fondations d'une école n'existe pas vraiment}

Dans ce sens il ne semble pas y avoir de grande continuité ou même de maintien dans les courants de pensée. Cette structure nécessaire qui formerait les fondations d'une école n'existe pas vraiment. Il y a cependant quelques exceptions. Ainsi, l'un des derniers ouvrages de M. Polèse et de R. Shearmur (Polèse et Shearmur 2002) donne une très bonne idée de ce qu'est une réalisation intellectuellement autonome et non dépendante même informellement d'un pouvoir public. L'ouvrage est libre, innovant et prospectif.

À cette étape, il serait intéressant de brosser rapidement quelques comparaisons avec nos homologues d'Amérique du Nord ou d'Europe. Les écoles américaines, celles des 
USA ou les écoles européennes, semblent en meilleure place dans cette notion d'école

Cette volonté d'être utile et visible du terrain a toujours été présente au Québec une perception de discipline indécise qui peut même sembler se contredire parfois. structurée. Aux États-Unis, les grands penseurs que furent entre autres W. Leontief, Walter Isard ou H.W. Richardson ont donné à cette discipline des sciences régionales des directions très claires qui se sont mues tout naturellement en véritable école.

En France des penseurs tels que F. Perroux ou P. Aydalot ont eu le même effet. Les puristes et les administratifs ont su garder leur distance et leur indépendance tout en entretenant d'étroites relations entre eux. On peut dire sensiblement la même chose pour des pays où les structures régionales sont, au départ, très différentes comme l'Allemagne ou l'Espagne. Ces différentes écoles sont multiples et concernent aussi bien les sciences régionales en général que la science régionale propre à un secteur particulier. Ainsi par exemple on peut parler d'une école des localisations optimales quand on parle de "L'État isolé » de Von Thünen du milieu du XIX ${ }^{\text {ième }}$ siècle qui fut suivi d'une pléiade d'écrits tous dirigés dans une direction semblable et vers des objectifs communs (A. Weber, W. Christaller, A. Lösh ... A. Schärlig puis C. Ponsard ou J. Thisse).

\subsection{Au Québec, une école pas ordinaire}

La province de Québec ne possède pas de véritables écoles traditionnelles. Elle possède cependant une école où interviennent des scientifiques qui pratiquent davantage la régiologie que les sciences régionales classiques. Cette "école pratique » se situe constamment sur le terrain, elle pratique des analyses serrées, formule des diagnostiques et émet des recommandations. À court terme, les choses se tiennent, mais aussitôt que l'on prend quelques années en considération (moyen et long terme) elle donne rapidement
Mais pourtant... c'est normal et même souhaitable. Cette école pratique s'assume et ne craint pas de s'afficher même si ses couleurs évoluent constamment. Dans ce sens elle est peu ordinaire et ne ressemble pas du tout aux «grandes écoles» américaines ou européennes qui elles sont plus rigides et peutêtre plus stables. Cette stabilité ne nous caractérise effectivement pas. Nous ne formons pas un courant dominant qui s'identifie au développement régional, cependant les décideurs prennent connaissance de nos analyses et nos recommandations. Les décisions ultérieures peuvent en être affectées. Cette volonté d'être utile et visible du terrain a toujours été présente au Québec dans bien des disciplines académiques. La science régionale en se transformant en régiologie n'échappe pas à cette volonté. Cette discipline est d'abord et surtout horizontale, elle aborde bien des secteurs de la socio-économie. Cependant sa verticalité peu développée et son image de «terrain» lui confèrent cette allure plutôt indécise.

Au Québec si nous n'avons pas vraiment cette structure d'école il est clair que nous possédons beaucoup d'éléments nécessaires à son édification. Dans ce sens, ce que nous possédons probablement le plus fortement ce sont des scientifiques et des experts. En proportion de notre population, nous avons un nombre respectable de tous ces regroupements d'intellectuels. Nous avons beaucoup d'écrivains et de scientifiques dont l'expertise est reconnue au niveau international. Toutes les branches de cette discipline sont couvertes autant en géographie (aménagement, occupation des sols, etc.) qu'en socio-économie (localisation, disparités, pauvreté, richesse, etc.) 
Il serait peut-être indélicat de nommer ces gens, la liste est longue et le risque d'un oubli est grand. Bien sûr on ne peut pas ignorer ou oublier des gens comme Clermont Dugas ${ }^{10}$ (géographe), Fernand Martin ${ }^{11}$ (économiste) ou Mario Polèse ${ }^{12}$ (urbaniste et analyste régional). Mais malgré ces nombreuses sommités, il n'y a pas véritablement d'école scientifique dotée d'une structure franche (upfront) comme telle. Il y a principalement des lignes de pensée qui souvent suivent les actions gouvernementales et qui souvent leur ressemblent. Ces lignes sont éphémères et ne dessinent pas de tendances solides et durables. Nous avons au Québec de nombreuses institutions publiques ou privées qui abritent la

\section{Conclusion}

Ces quelques lignes confirment ce qui est avancé en introduction. Une école traditionnelle, selon les critères souvent retenus par d'autres pays? Non il n'y en a pas encore et il n'y en aura probablement jamais. D'accord, nous avons peut-être une école mais notre école n'est pas une école ordinaire. C'est une école qui possède peu de racines mais qui prend beaucoup d'espace sur le terrain, le concret. Souvent elle est utile et presque toujours, elle colle à la réalité.

Finalement il s'agit d'une école des sciences régionales qui chemine selon certains gènes que l'on retrouve bien souvent au Québec dans d'autres disciplines. Des gènes de grande liberté intellectuelle sans grande retenue. Des gènes où la réflexion accepte et même recherche très vite la validation que seul le terrain peut donner ou demander. Notre science régionale s'identifie alors davantage à la régiologie. Des gènes où l'extrême émerge facilement. Des gènes discipline. Ainsi, l'INRS à Montréal, le CRDT à Rimouski, et plusieurs laboratoires reconnus font progresser les connaissances dans toutes les directions. Ces institutions génèrent des thèses, élaborent des manuels académiques, publient des revues savantes et des ouvrages collectifs, diffusent des contributions scientifiques internationales et multiplient les colloques et congrès. Elles participent aussi à l'avancement des connaissances, à la formation d'experts et parfois à l'orientation des politiques publiques. Avons-nous pour autant une véritable école de sciences régionales?

d'autonomie marquée, la science régionale québécoise reste indépendante et autosuffisante vis-à-vis de ses équivalentes américaine ou européenne. Bien sûr dans un tel contexte, les «valses» et les retournements sont fréquents mais chaque période enrichit la suivante. Dans ce sens notre discipline est sur additive, la somme des acquis globaux dépasse largement la somme des acquis de chaque période individuelle. Des positions extrêmes ont été analysées au cours des années '60. Elles ont été décriées et rejetées par la suite, mais elles ont tout de même apporté quelques pierres à l'édifice. Dans ce sens notre discipline s'est toujours assumée avec franchise et honnêteté même si elle reste bien indécise. 


\section{Notes et références}

${ }^{1}$ Jean-Claude Lebel, 1965

${ }^{2}$ François Perroux n'était pas un économiste régional mais, entre autres, un économiste industriel. De grandes notions telles que celles des pôles de développement se retrouvent dans Perroux (1955)

${ }^{3}$ Walter Isard a publié 21 livres sur ce sujet depuis la fin des années 40

${ }^{4}$ Joanis, Martin, St-Cernis, 2004, p.33

${ }^{5}$ La DATAR a été remplacée il y a quelques années par un organisme similaire, la DIACT

${ }^{6}$ Higgins, Martin et Reynaud, 1970

${ }^{7}$ C. Dugas, «Les régions périphériques », 1983, p.67

${ }^{8}$ Voir particulièrement Martin (2002), p. 43

${ }^{9}$ Voir par exemple Polèse et Shearmur 2002 ou Proulx 2002

${ }^{10}$ Voir Dugas (1983)

${ }^{11}$ Voir par exemple la production à caractère historique : Joanis, Martin, St-Cernis, (2004)

${ }^{12}$ Voir par exemple Polèse (1999)

Dugas, C. (1983). Les régions périphériques, défi au développement du Québec, Presse de l'Université du Québec

Gendron, F. (1983). Le choix des régions : document de consultation sur le développement des régions, Montréal : Office de planification et de développement du Québec.

Gravel, J.-P. (1999), Retour sur l'évolution régionale au Québec, monographie, Groupe Urbatique

Hamelin L.-E. (2006), L’âme de la terre : parcours d’un géographe, Éditions Multi Mondes.

Higgins, B., F.Martin, et A. Reynaud (1970). Les orientations du développement économique régional dans la province de Québec, Ottawa : Ministère de l'expansion économique régionale

Joanis M., F. Martin et S. St-Cernis (2004). Quel avenir pour les politiques de développement régional au Québec, CIRANO, version révisée.

Lebel, J.-C. (1965), Le BAEQ: une voie d'approche originale au problème des disparités régionales, monographie, s.éd., 23 p.

Martin, F. (2002). «Un schéma de croissance pour la Rive - Sud », étude commandée par la Commission Nicolet, disponible sur le site Internet www.mobilité-mtl-rsud.gouv.qc.ca/publications/

Perroux, F. (1955). « Note sur la notion de pôle de croissance ». Économie appliquée, 8: 307-320.

Picotte, Y. (1992). Développer les régions du Québec, Secrétariat aux affaires régionales,

Gouvernement du Québec.

Polèse, M. (1999). «From Regional Development to Local Development: On the Life, Death and Rebirth of Regional Science As a Policy Relevant Science », Revue canadienne des sciences régionales, vol. XXII, no. 3, pp. 299-314.

Polèse, M. et R. Shearmur (2002). La périphérie face à l'économie du savoir : la dynamique spatiale de l'économie canadienne et l'avenir des régions non métroplitaines du Québec et des Provinces de l'Atlantique, INRS-UCS et ICRD, Montréal et Moncton, 2002. http:/www.inrs-ucs.uquebec.ca/default.asp? $p=r e s$

Proulx, M.-U. (2002). L'économie des territoire au Québec, Sainte-Foy : Presses de 1’Université du Québec.

Rapport Parent (1963 - 1964), 5 tomes, Rapport de la commission royale d'enquête sur l'enseignement dans la province de Québec. Québec : Gouvernement du Québec. 
Publicité MAMR 